\title{
Beyond the isobar model: Rescattering in the system of three particles.
}

\author{
Mikhail Mikhasenko* and Bernhard Ketzer \\ Universität Bonn, Helmholtz-Institut für Strahlen- und Kernphysik, 53115 Bonn, Germany \\ E-mail: mikhasenko@hiskp.uni-bonn. de, ketzer@hiskp.uni-bonn. de
}

\begin{abstract}
The isobar model is an essential part of partial wave analyses (PWA) of multi-body hadronic final states. In the isobar model, all final states are described as sequential two-body decays of intermediate resonances. It turned out to work surprisingly well in the past, despite the fact that it neglects rescattering, i.e. interactions in the final state. With the advent of data samples of extremely high statistical significance from ongoing experiments, effects due to final state interactions may become important in order to understand the data. We discuss the example of the recently observed $a_{1}(1420)$ in the $3 \pi$ final state using PWA techniques. In our model, we interpret the signal as a result of rescattering in the final state. In the presence of coupled $\pi \pi \pi$ / $K \bar{K} \pi$ channels, final-state interaction causes a redistribution of events between the $\pi \pi \pi$ and $K \bar{K} \pi$ systems. We compare our interpretation to the genuine resonance hypothesis and discuss a sophisticated approach to clarify the nature of the signal.
\end{abstract}

54th International Winter Meeting on Nuclear Physics

25-29 January 2016

Bormio, Italy

* Speaker. 


\section{Introduction}

The goal of hadron spectroscopy is to identify resonances and to identify their nature, e.g. ordinary bound states of quarks and antiquarks or exotic states with different degrees of freedom. The states are accessible in a scattering experiment. An isolated narrow resonance appears in the spectrum as a peak, the phase difference runs rapidly through $90^{\circ}$, with a total phase motion of $180^{\circ}$. But usually the experimentally measured spectrum is more complicated than a single isolated resonance. The reasons and consequences are as follows:

- The first difficulty is due to wide and overlapping resonances. Generally, amplitudes for different resonances interfere, changing the shape of the observed intensity and the corresponding phase motion. A coherent sum of two resonance amplitudes (e.g. Breit-Wigner) is not the correct answer for the amplitude in presence of two resonant poles.

- The two-body system is the simplest scattering system. The experimental and theoretical understanding of low partial waves for the $\pi \pi$ and $\pi K$ systems has advanced considerably over the last 30 years. Unfortunately, we still do not know much about many other two-body systems $\left(\pi \eta, \pi \eta^{\prime}, \pi \omega, \ldots\right)$. The behavior of amplitudes in the high mass region $>1.5 \mathrm{GeV}$ is not well known and parameterized.

- The system of three interacting particles is very rich because, on the one hand, resonances appear in pair-interactions as well as in the full dynamics of the system. On the other hand, however, scattering effects and inelastic channels may enhance observables in a similar way as resonances $[1,2,3,4,5]$.

In the first section of this paper we discuss the recent observation of a tiny but very interesting enhancement in $3 \pi$ final state, the $a_{1}(1420)$ [6]. We treat the observation as a rescattering effect caused by a peculiar kinematics and the presence of the $K K \pi$ channel coupled to $3 \pi$. We calculate the diagram for the first order rescattering and compare two different model fits to the COMPASS data on the $a_{1}(1420)$ : our rescattering model vs. the Breit-Wigner model presented in [6]. The second section is dedicated to a systematic approach in the theory of rescattering. We demonstrate the application of general scattering theory to the three-body final state. We set up the KhuriTreiman equations [7] for the scalar amplitude with two subchannels with attractive resonance interactions and solve them numerically. Finally, we discuss their interpretation.

\section{Resonance-like behaviour of non-resonant amplitude}

The $a_{1}(1420)$ is a resonance-like signal observed by the COMPASS collaboration [6] and confirmed by the VES collaboration [8]. A partial wave analysis in bins of the $3 \pi$-mass and the reduced squared 4-momentum transfer $t^{\prime}$ has been employed by COMPASS to extract the intensity and the relative phase motions for 88 -waves. An unexpected resonance-like behavior has been observed in the $1^{++} f_{0}(980) \pi P$-wave near $1.4 \mathrm{GeV}$. A clear peak structure and a rapid phase motion with respect to the $1^{++} \rho \pi S$-wave suggest the presence of a new resonance in the $J^{P C}=1^{++}$sector different to the well-known $a_{1}(1260)$. The signal was called $a_{1}(1420)$.

There are several issues which make the observation of this structure extremely interesting: 
- The $a_{1}(1420)$ can not be a radial excitation of the $a_{1}(1260)$ firstly because the peak is too close to the ground state. Secondly, the width of the $a_{1}(1420)$ is much smaller than the width of the $a_{1}(1260)$ which contradicts the expectation. Thirdly, the $a_{1}(1420)$ does not fit to the radial excitation trajectory.

- The $a_{1}(1420)$ does not appear in lattice calculations [9].

- The $a_{1}(1260)$ is allowed to decay to the $1^{++} f_{0}(980) \pi P$-wave. Then the intensity of this wave is expected to grow rapidly from the $f_{0}(980) \pi$ threshold on the tail of the wide $a_{1}(1260)$. This feature is not observed.

- The position of the peak almost coincides with the threshold for the $K^{\star} \bar{K}$ channel.

We suggest a dynamic explanation of the phenomenon which does not require a genuine resonance [2]. A final state interaction is considered for the coupled-channels system $1^{++} f_{0}(980) \pi P$ wave and $1^{++} K^{\star} \bar{K} S$-wave. We find that cross-channel scattering $K^{\star} \bar{K} \rightarrow f_{0}(980) \pi$ via $K$ exchange has a very peculiar resonance-like behavior exactly at the mass of the observed $a_{1}(1420)$ signal.

First we give a kinematical explanation of the phenomenon. We consider the decay of the broad $a_{1}(1260)$ with invariant mass $w$ to real $K^{\star}$ and $\bar{K}$ in the rest frame. They fly back to back with the same momentum. When the $K^{\star}$ subsequently decays to real $K \pi$, it may happen that the $K$ goes to the same direction as $\bar{K}$ with a velocity higher than the $\bar{K}$, and thus catching up with it. The invariant mass of the $K \bar{K}$ system is determined by $w$. The invariant mass of collinear $K \bar{K}$ is precisely the mass of $f_{0}(980)$ when $w=1.42 \mathrm{GeV}$.

Mathematically the resonance-like behavior is caused by a logarithmic branch point on the second Riemann sheet close to the physical region. Whatever the production mechanism of $K^{\star} \bar{K}$ is, the amplitude for the $f_{0} \pi$ final state via rescattering is given by a loop integral. It might be a triangle loop if the kaon system originates from $a_{1}(1260)$ non-resonant production. The loop integral has a singularity for the peculiar kinematics discussed above. The effect is known as triangle singularity because the branch point $a_{1}(1420)$ is made by the leading singularity of the triangle diagram. Landau classified the singularities of loop integrals [10]. Coleman and Norton [11] formulated the necessary conditions for singularities in loops. They found that singularities of loop integrals correspond to classical scattering processes with all particles on mass shell.

The rescattering process being enhanced by the triangle singularity leads to a migration of events from the $K K \pi$ final state to the $3 \pi$ final state (a general discussion can be found in Ref. [12]). To demonstrate this idea we show in Fig. 1 the kinematically allowed region of the Dalitz plot for the $\pi^{+} \pi^{-} \pi^{-}$system superimposed by the one for $K \bar{K} \pi$. The invariant mass squared of the $\pi^{+} \pi^{-}$ or $K \bar{K}$ subsystem is along the $x$ axis, the one of the other $\pi^{+} \pi^{-}$or the $K \pi$ subsystem is along the $y$ axis, for the $3 \pi$ or $K \bar{K} \pi$ system, respectively. The horizontal dashed line indicates the $K^{\star}$ resonance, the vertical dashed line shows the position of the $f_{0}(980)$ resonance for both kaon and pion subsystems. When we change the invariant mass of the full system $w$, the sizes of the Dalitz plots change and consequently the positions of their borders with respect to the indicated resonances. When $w=1.4 \mathrm{GeV}$ the intersection of the dashed lines coincides with the border of the kaon Dalitz plot, i.e. the 3-momentum vectors of the decay products are aligned. In this case, events are redistributed from the intersection point along the $f_{0}(980)$ vertical dashed line. Since $f_{0}$ 


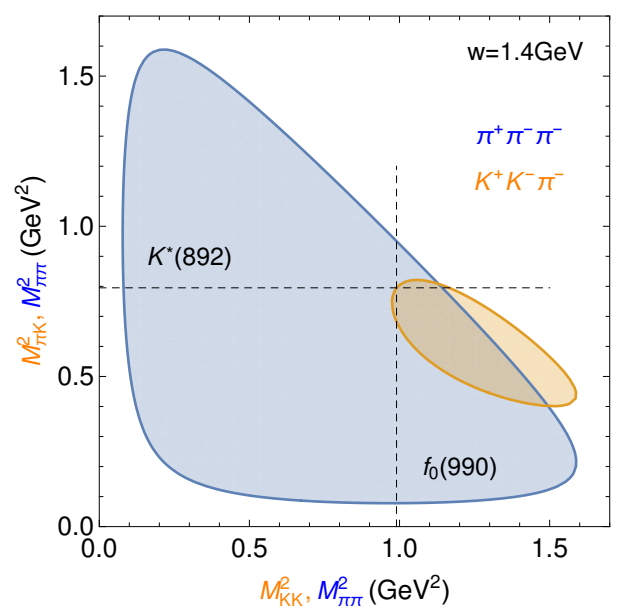

Figure 1: Kinematically allowed regions of the Dalitz plots for (blue) the $3 \pi$ and (orange) the $K \bar{K} \pi$ systems at $w=1.4 \mathrm{GeV}$. The position of the $f_{0}(980)$ resonance is shown by the vertical dashed line. The horizontal dashed line indicates the $K^{\star}$ resonance for the $K \pi$ subsystem.

has both a $\pi \pi$ and a $K \bar{K}$ decay channel, this results in a migration of events from the $K \bar{K} \pi$ Dalitz plot to the $3 \pi$ Dalitz plot [12].

To compare the hypothesis of a new resonance to our explanation we perform a fit of the intensities of the $1^{++} \rho(770) \pi S$-wave, the $1^{++} f_{0}(980) \pi P$-wave and their relative phase motion for one $t^{\prime}$ bin. We use the resonance model of Ref. [6], where the amplitude for every wave is a sum of a non-resonance background term (flexible curve) and a Breit-Wigner signal part. For our alternative interpretation we use the rescattering amplitude as input for the signal in the $1^{++} f_{0}(980) \pi P$-wave. As a result, the $1^{++} \rho(770) \pi S$-wave is fitted similarly well in our model, the result being similar to Fig. 3a of Ref. [6]. Here we show the fit results for the $1^{++} f_{0}(980) \pi P$-wave in the representation of the Argand diagram.

The left side of Fig. 2 shows the fit with the rescattering amplitude for the signal, the right plot is the fit with the resonance (Breit-Wigner) parameterization of the signal part of $1^{++} f_{0}(980) \pi P-$ wave. For both fits the signal component is shown as a blue line; it determines the main phase motion. The non-resonant background has no phase motion but, coherently contributing to the amplitude, it shifts every point of the blue curves. The red curves shows the full amplitude as a result of the fit.

The rescattering amplitude (blue curve on the left plot of Fig. 2) develops a phase motion over more than $180^{\circ}$. A part of the phase motion is due to the wide $a_{1}(1260)$ resonance which decays to $K^{\star} K$. The rapid phase motion in the interval of the fit is related to the triangle singularity. In the Breit-Wigner hypothesis the signal curve (blue curve on the right side of Fig. 2) is a circle by construction.

Note that the Breit-Wigner amplitude has two free parameters, the mass and the width. The rescattering amplitude is completely fixed and has no free parameters. We conclude that the COMPASS data are equally well described by both models, with similar $\chi^{2}$.

Our model of the final state interaction is still very simplified. To make a solid statement about the nature of $a_{1}(1420)$ a more sophisticated analysis of the rescattering processes has to be done. The next section is dedicated to the current progress in this direction. 

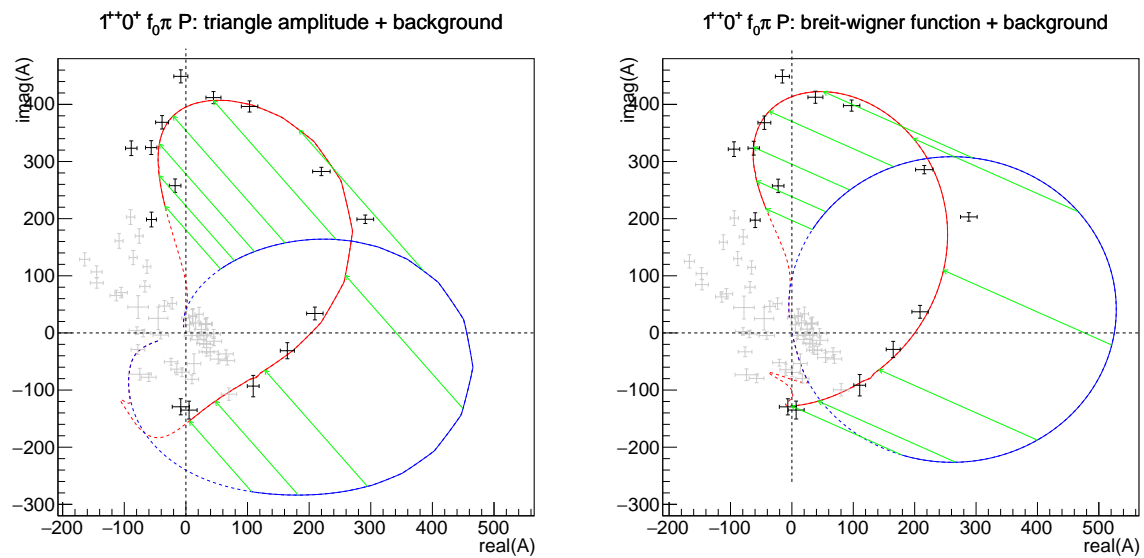

Figure 2: Argand diagram for $1^{++} f_{0}(980) \pi P$. The phase is measured with respect to $1^{++} \rho(770) \pi S$. Two hypotheses are tested: the left plot is the result of the fit with the rescattering amplitude to describe the signal, the right plot the result with the Breit-Wigner parameterization of the signal. The data points are the result of the PWA in mass and $t^{\prime}$ bins preformed by COMPASS. The ones printed in black have been used for both fits while the grey ones are further away from the signal region and have not been taken into account. The full amplitude from the fit of the intensities of the $1^{++} f_{0}(980) \pi P$ and $1^{++} \rho(770) \pi S$ waves and their relative phase is shown as red curve. The signal contribution is shown in blue for both fits, the non-resonant background by green arrows. It does not exhibit a phase motion but shifts the amplitude for both fits.

\section{Systematic approach to rescattering}

One may notice that the triangle diagram is only first term of the full rescattering series. This brings up further questions:

- How close is the first term to the full process taking into account all possible rescatterings?

- Is there a method to sum up the full rescattering series?

- Does it converge?

The approach which we consider in the following has been suggested in Ref. [7] and is often called Khuri-Treiman equations. It employs the principle of unitarity to answer the questions above. To demonstrate the approach we consider a simple example, which is unphysical because of its simplifications, but serves as an illustration of the method. Let $A=A\left(s_{1}, s_{2}, s_{3}\right)$ be the amplitude for the $\pi_{1} \pi_{2} \pi_{3}$ final state, where $s_{1}=\left(p_{2}+p_{3}\right)^{2}, s_{2}=\left(p_{3}+p_{1}\right)^{2}, s_{3}=\left(p_{1}+p_{2}\right)^{2}$. We assume that the $\pi_{i}, i=1 \ldots 3$ are distinguishable scalar particles with 4-momenta $p_{i}$ and known interaction amplitudes at fixed $w$. The amplitude for $\pi_{2} \pi_{3}$ scattering is $t^{(1)}\left(s_{1}\right)=\left\langle\pi_{2} \pi_{3}|\hat{T}| \pi_{2} \pi_{3}\right\rangle$. Analogously, the scattering amplitude for $\pi_{1} \pi_{2}$ is $t^{(3)}$. In this example, we use a parameterization of the $(\pi \pi)$ $S$-wave fitted to Ref. [13] for $t^{(1)}$, and a $\rho$-like Breit-Wigner amplitude for $t^{(3)}$, for simplicity with the two pions in an $S$-wave. We make a further simplification by setting interaction amplitude for $\pi_{1} \pi_{3}$ scattering to zero.

We know that whatever the answer for the full amplitude $A$ is, it must satisfy elastic unitarity:

$$
\Delta_{s_{i}} A=2 i \int A \mathrm{~d} \rho_{i} t^{(i) *}, \quad i=1 \ldots 3 .
$$


where $\Delta_{s_{i}} A$ is the discontinuity of the amplitude $A$ across the unitarity cut in the two-body scattering channel $i$ (i.e. $\pi_{2} \pi_{3}$ for $i=1$, etc.), $\rho_{i}$ is a half of the phase-space of intermediate two-body states for the corresponding channel.

A first guess at the expression for the full amplitude of this example may be

$$
A\left(s_{1}, s_{3}\right)=c^{(1)} t^{(1)}\left(s_{1}\right)+c^{(3)} t^{(3)}\left(s_{3}\right),
$$

where the $c^{(i)}$ are production constants which do not depend on $s_{i}$ but on $w^{2}$ only, representing the dynamics of the full system (three-particle resonances). This representation is a standard ansatz for the PWA used by many experimental groups. The approach is called the Isobar Model (IM).The problem is this parameterization does not satisfy Eq. 3.1.

In the Khuri-Treiman approach, we construct the full amplitude for the problem in a similar way as the sum of amplitudes for every subchannel:

$$
A\left(s_{1}, s_{3}\right)=A^{(1)}\left(s_{1}, s_{3}\right)+A^{(3)}\left(s_{3}, s_{1}\right), \text { with } \quad A^{(i)}=\sum_{l}(2 l+1) a_{l}^{(i)} P_{l}\left(z_{i}\right),
$$

where $a_{l}^{(i)}$ denotes the two-body partial wave of angular momentum $l$ (corrected for rescattering), $P_{l}\left(z_{i}\right)$ the Legendre polynomials and $z_{i}=\cos \theta_{i}$ with $\theta_{i}$ the scattering angle in the subchannel $i$. Keeping only terms with $l=0$, the expression for $a_{0}^{(1)}$ satisfying elastic unitarity is [7]

$$
a_{0}^{(1)}\left(s_{1}, s_{3}\right)=t_{0}^{(1)}\left(s_{1}\right)\left[c_{0}^{(1)}+\frac{1}{\pi} \int \mathrm{d} s^{\prime} \frac{\rho_{1}\left(s^{\prime}\right) b_{0}^{(1)}\left(s^{\prime}\right)}{s^{\prime}-s_{1}}\right],
$$

with

$$
b_{0}^{(1)}\left(s_{1}\right)=\int \frac{\mathrm{d} z_{1}}{2} a_{0}^{(3)}\left(s_{3}\left(s_{1}, z_{1}\right), s_{1}\right)
$$

being the projection to the subchannel amplitude from the crossed subchannels. The corresponding amplitude $a_{0}^{(3)}$ is constructed analogously by the replacement $3 \leftrightarrow 1$.

The result is a system of coupled integral equations, which can be solved numerically following Ref. [14]. Since the equations depend linearly on the couplings $c_{0}^{(i)}$, the solution can be cast in the form

$$
A=\left(\alpha_{\text {direct }}^{(1)}+\alpha_{\text {induced }}^{(3)}\right) c_{0}^{(1)}+\left(\alpha_{\text {direct }}^{(3)}+\alpha_{\text {induced }}^{(1)}\right) c_{0}^{(3)},
$$

where $\alpha_{\text {direct }}^{(1)} \equiv a_{0}^{(1)}, \alpha_{\text {induced }}^{(3)} \equiv a_{0}^{(3)}$ are solutions of the equations with $c_{0}^{(1)}=1, c_{0}^{(3)}=0$, and $\alpha_{\text {direct }}^{(3)} \equiv a_{0}^{(3)}, \alpha_{\text {induced }}^{(1)} \equiv a_{0}^{(1)}$ are solutions of the equations with $c^{(1)}=0, c^{(3)}=1$. A comparison between the moduli of $t_{0}^{(i)}, \alpha_{\text {direct }}^{(i)}$, and $\alpha_{\text {induced }}^{(i)}$ are shown in Fig. 3 for both amplitudes $i=1,3$, with $t_{0}^{(1)}$ representing a $(\pi \pi) S$-wave and $t_{0}^{(3)}$ a $\rho$-like signal, as mentioned above.

The meaning of Eq. 3.6 is as follows. Consider the part of the amplitude which corresponds to the production of the interacting pair $\pi_{2} \pi_{3}$. Without rescattering this amplitude would be $t_{0}^{(1)}$. Rescattering with the bachelor pion $\pi_{1}$ results in a projection of this amplitude onto the crossed channel $\pi_{1} \pi_{2}$. A non-zero amplitude for the crossed-channel interaction in turn reflects back and changes the shape of the initial amplitude. The resummation of all scattering processes where the last scattering is the same as the first carries index $\alpha_{\text {direct }}$ while the reflection terms are denoted as $\alpha_{\text {induced }}$ 

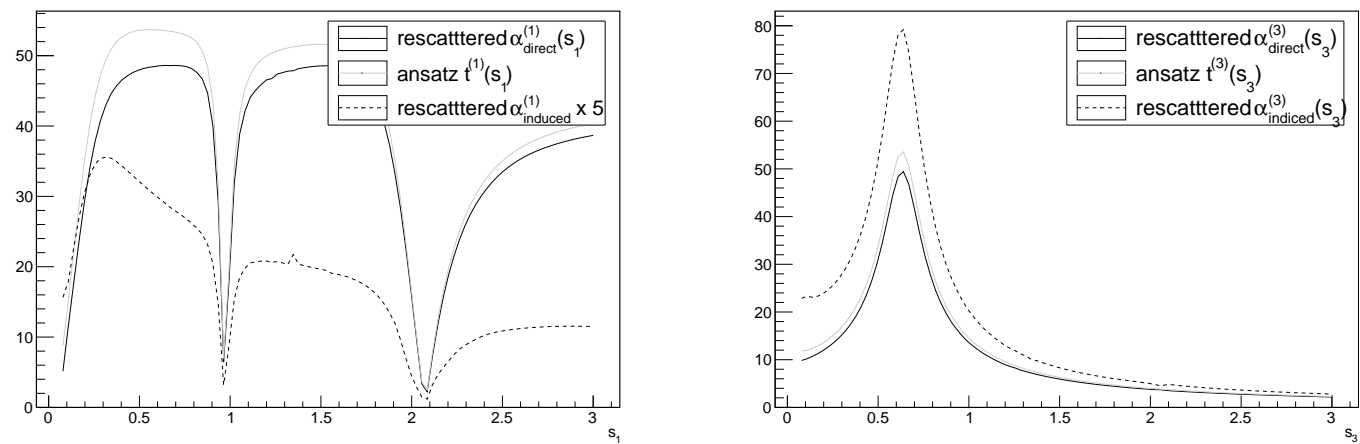

Figure 3: Modification of the isobar shapes (here: moduli of amplitudes) due to rescattering. The gray curves demonstrate the ansatz for the amplitudes $t_{0}^{(i)}$ in the Khuri-Treiman equations. The modified shapes of these amplitudes in the direct channels are shown by black solid lines, the induced shapes are shown by black dashed lines. See text for further details.

This approach has already been successfully applied to several reactions [15, 16, 17, 14]; the complexity of the model is currently being increased continuously. The first two items summarize the recent achievements, the further items represent our ideas to proceed:

1. The $3 \pi$ final state from $\omega, \phi$ decays has only one major subchannel amplitude $\left(\pi^{+} \pi^{-}\right)_{P}$ i.e. the $\rho(770)$.

2. The analysis of the $K^{-} \pi^{+} \pi^{+}$final state produced in $D^{+}$decays performed in Ref. [14] is more complicated because it includes 8 scattering amplitudes.

3. The decay $K \rightarrow 3 \pi$ was one of the original motivation for Khuri-Treiman equations. With the recent precise data from NA48/2 experiment it becomes interesting again to apply the formalism because of the prominent peak in the $\pi^{0} \pi^{0}$ spectrum [18]. It is the reaction where the extension of the formalism to the coupled-channel case can be tested.

4. The $\pi \pi \pi$ and $\pi K K$ final states from $\tau$ decay have fixed quantum numbers $J^{P C}=1^{++}$. The biggest data set is collected by CLEO Collaboration [19]. The invariant mass of the system $w$ runs from threshold to $1.8 \mathrm{GeV}$. To describe the reaction one indeed needs to have a model for the dynamics of the full system, which is not part of the approach considered here. An alternative way to proceed is a mass-independent analysis, widely used by the COMPASS and VES experiments [20]. The idea here is to perform the Khuri-Treiman analysis independently in bins of $w$ without any assumptions on $c^{(i)}(w)$ (essentially constants at every bin). The study of the dynamics in $w$ is then considered as a second step of the analysis.

This approach could clarify the $a_{1}(1420)$ puzzle discussed above. The difficulties are the following: many waves have to be considered, a coupled-channels formalism is required, taking into account spin is essential because it results in several waves for a given spin for $\pi \pi$ subsystem (e.g. $\rho \pi S$-wave and $\rho \pi D$-wave).

5. The diffractively produced $3 \pi$ final state has the highest complexity among the available data samples. In addition to the wide $3 \pi$ spectrum, the quantum numbers $J^{P C}$ of the final 
system are not fixed. The main PWA model used by COMPASS consist of 88 waves, with $J^{P C}$ sectors $0^{-+}, 1^{++}, 2^{++}, 2^{-+}, \ldots$ It describes the data very well while the interpretation of the extracted $w$-dependence is model-dependent, e.g. by fixing the isobar shapes.

The method we intend to approach the complexity of the problem is to replace the classical partial waves by Khuri-Treiman partial waves, i.e. the expressions in the parentheses of Eq. 3.6, in one specific sector, e.g. $0^{-+}$, while keeping the other sectors filled with standard partial waves.

It is worth noticing that low two-body partial waves relevant for the problem are well constrained $[13,21]$.

\section{Summary and outlook}

Understanding the interactions in a three-body system is a modern challenge in spectroscopy. Many obstacles to this task in the past have been overcome: very precise data have been collected, computational resources are highly developed. We are now fully equipped to tackle the problem of the three-body system.

In this context, the $a_{1}(1420)$ is a unique object. It appears in the system of the three lightest hadrons. As we discussed, it is likely related to the effect of three-body final state interaction which makes a difference to two-body system. The inelastic channel with a rather narrow resonance and the peculiar kinematics mimic a resonance behavior. We have a solid method to approach the problem and we are on the way to solve it. Similar mechanisms might play a very important role in the charmonium and bottomonium sector.

\section{References}

[1] N. N. Achasov and A. A. Kozhevnikov, On the Nature of C (1480) Resonance, Z. Phys. C 48 (1990) $121-130$.

[2] M. Mikhasenko, B. Ketzer and A. Sarantsev, Nature of the $a_{1}$ (1420), Phys. Rev. D 91 (2015) 094015, [1501.07023].

[3] A. P. Szczepaniak, Triangle Singularities and XYZ Quarkonium Peaks, 1501.01691.

[4] F.-K. Guo, U.-G. Meissner, W. Wang and Z. Yang, How to reveal the exotic nature of the $P_{c}(4450)$, Phys. Rev. D 92 (2015) 071502, [1507.04950].

[5] M. Mikhasenko, A triangle singularity and the LHCb pentaquarks, 1507.06552.

[6] COMPASS collaboration, C. Adolph et al., Observation of a New Narrow Axial-Vector Meson $a_{1}$ (1420), Phys. Rev. Lett. 115 (2015) 082001, [1501.05732].

[7] N. N. Khuri and S. B. Treiman, Pion-Pion Scattering and $K^{ \pm} \rightarrow 3 \pi$ Decay, Phys. Rev. 119 (1960) $1115-1121$.

[8] VeS Collaboration. collaboration, Y. Khokhlov, V. Dorofeev, A. Ekimov, V. Gotman, A. Ivashin et al., Partial wave analyses of the $\pi^{-} \pi^{0} \pi^{0}$ and $\pi^{-} \pi^{0}$ systems with VES setup, PoS Hadron2013 (2014) 088. 
[9] J. J. Dudek, R. G. Edwards, B. Joo, M. J. Peardon, D. G. Richards et al., Isoscalar meson spectroscopy from lattice QCD, Phys. Rev. D 83 (2011) 111502, [1102.4299].

[10] L. Landau, On analytic properties of vertex parts in quantum field theory, Nucl. Phys. 13 (1959) 181-192.

[11] S. Coleman and R. E. Norton, Singularities in the physical region, Nuovo Cim. 38 (1965) 438-442.

[12] A. P. Szczepaniak, Dalitz plot distributions in presence of triangle singularities, Phys. Lett. B757 (2016) 61-64, [1510.01789].

[13] R. Garcia-Martin, R. Kaminski, J. R. Pelaez, J. Ruiz de Elvira and F. J. Yndurain, The Pion-pion scattering amplitude. IV: Improved analysis with once subtracted Roy-like equations up to $1100 \mathrm{MeV}$, Phys. Rev. D83 (2011) 074004, [1102.2183].

[14] F. Niecknig and B. Kubis, Dispersion-theoretical analysis of the $D^{+} \rightarrow K^{-} \pi^{+} \pi^{+}$Dalitz plot, JHEP 10 (2015) 142, [1509.03188].

[15] F. Niecknig, B. Kubis and S. P. Schneider, Dispersive analysis of $\omega \rightarrow 3 \pi$ and $\phi \rightarrow 3 \pi$ decays, Eur. Phys. J. C72 (2012) 2014, [1203. 2501 ].

[16] I. V. Danilkin, C. Fernandez-Ramirez, P. Guo, V. Mathieu, D. Schott, M. Shi et al., Dispersive analysis of $\omega / \phi \rightarrow 3 \pi, \pi \gamma *$, Phys. Rev. D 91 (2015) 094029, [1409.7708].

[17] P. Guo, I. V. Danilkin, D. Schott, C. Fernandez-Ramirez, V. Mathieu and A. P. Szczepaniak, Three-body final state interaction in $\eta \rightarrow 3 \pi$, Phys. Rev. D 92 (2015) 054016, [1505.01715].

[18] NA48/2 collaboration, J. R. Batley et al., Observation of a cusp-like structure in the piO piO invariant mass distribution from $\mathrm{K+-} \longrightarrow$ pi+- piO piO decay and determination of the pi pi scattering lengths, Phys. Lett. B633 (2006) 173-182, [hep-ex/ 0511056$].$

[19] CLEO Collaboration collaboration, D. Asner et al., Hadronic structure in the decay $\tau^{-} \rightarrow v_{\tau} \pi^{-} \pi^{0} \pi^{0}$ and the sign of the tau-neutrino helicity, Phys. Rev. D 61 (1999) 012002, [hep-ex/9902022].

[20] C. Adolph et al., Resonance Production and $\pi \pi S$-wave in $\pi^{-}+p \rightarrow \pi^{-} \pi^{-} \pi^{+}+p_{\text {recoil }}$ at 190 GeV/c, 1509.00992.

[21] P. Buettiker, S. Descotes-Genon and B. Moussallam, A new analysis of $\pi K$ scattering from Roy and Steiner type equations, Eur. Phys. J. C 33 (2004) 409-432, [hep-ph/ 0310283 ]. 